\title{
COMPOSTOS ANTIOXIDANTES EM FRUTOS DE ACESSOS DE CARAMBOLEIRA EM DIFERENTES AMBIENTES DE PERNAMBUCO'
}

\author{
JOSÉ SEVERINO DE LIRA JÚNIOR², JOÃO EMMANOEL FERNANDES BEZERRA², \\ ILDO ELIEZER LEDERMAN ${ }^{4}$, LÍDIA CRISTINA DOS SANTOS ALENCAR CORREIA ${ }^{5}$, \\ MARIA INÊS SUCUPIRA MACIEL ${ }^{6}$
}

RESUMO - A polpa da carambola contém compostos químicos com propriedades antioxidantes importantes à saúde humana, contra o envelhecimento celular e a prevenção de doenças. Este trabalho objetivou avaliar o fruto de acessos de caramboleira quanto às concentrações de compostos antioxidantes. Foram analisadas as concentrações de ácido ascórbico, carotenoides totais, flavonoides, fenólicos totais e taninos em cinco acessos selecionados de carambola, cultivada em três distintas regiões edafoclimáticas de Pernambuco. Os acessos de carambola diferiram significativamente entre si, quanto às concentrações de ácido ascórbico, fenólicos totais e taninos. A significância para os efeitos de ambiente confirmou que os locais de cultivo são heterogêneos, em relação ao potencial produtivo de frutos com diferentes concentrações médias de ácido ascórbico, flavonóis, fenólicos totais e taninos. A interação significativa entre genótipos e ambientes indica que os acessos de carambola apresentam respostas diferenciadas às concentrações de ácido ascórbico, flavonóis, fenólicos totais e taninos, quando cultivadas em diferentes locais. As condições de cultivo da Estação Experimental do IPA Itambé e os acessos IPA-7.2, IPA-22.3 proporcionaram as maiores concentrações de ácido ascórbico, fenólicos totais e taninos.

Termos para indexação: Oxalidaceae, fruta exótica, alimento funcional.

\section{ANTIOXIDANT COMPOUNDS IN FRUITS OF Averrhoa carambola ACCESSIONS UNDER DIFFERENT ENVIRONMENTS IN THE STATE OF PERNAMBUCO, BRAZIL}

\begin{abstract}
The star fruit pulp contains chemical compounds with antioxidant properties important to human health, against cellular aging and disease prevention. This study aimed to evaluate the fruit of Averrhoa carambola accessions regarding the concentrations of antioxidant compounds. It was analyzed the concentrations of ascorbic acid, total carotenoids, flavonoils, total phenolics and tannins in five star fruit accessions, grown in different edaphoclimatic conditions of the state of Pernambuco. The star fruit accessions differed significantly among themselves regarding the concentrations of ascorbic acid, total phenolics and tannins. The significance for the environment effects confirmed that the cultivation sites are heterogeneous in relation to the productive potential of fruits with different average concentrations of ascorbic acid, flavonoils, phenolic compounds and tannins. A significant interaction between genotypes and environments indicates that star fruit selections exhibit differential responses to ascorbic acid, flavonoils, total phenolics and tannins, when grown in different sites. The experimental Station IPA Itambe's cultivation conditions and IPA-7.2, IPA-22.3 accessions showed the highest concentrations of ascorbic acid, total phenolics and tannins.

Index terms: Oxalidaceae, exotic fruit, functional food.
\end{abstract}

\footnotetext{
${ }^{1}$ (Trabalho 306-13). Recebido em: 26-08-2013. Aceito para pubilcação em: 04-08-2014.

${ }^{2}$ Eng. Agr., D.Sc., Pesquisador do Instituto Agronômico de Pernambuco - IPA. E-mail: lira.junior@ipa.br

${ }^{3}$ Eng. Agr., M.Sc., Pesquisador do Instituto Agronômico de Pernambuco - IPA. E-mail: joao.emmanoel@ipa.br

${ }^{4}$ Eng. Agr., PhD., Pesquisador da Embrapa-IPA aposentado. E-mail: ildo@ipa.br

${ }^{5}$ Ms.C em Ciência e Tecnologia de Alimentos-UFRPE, E-mail: aidil_alencar@yahoo.com.br

${ }^{6}$ D.Sc., Professora do Departamento de Ciência e Tecnologia de Alimentos da UFRPE, E-mail: m.ines@dcd.ufrpe.br
} 


\section{INTRODUÇÃO}

A caramboleira Averrhoa carambola L. é uma fruteira tropical do Sudeste asiático, cultivada na Malásia, sul da China, Taiwan e Índia, sendo relativamente popular nas Filipinas, Austrália e em algumas ilhas do Pacífico Sul (DONADIO et al., 2001).

No Brasil, esta fruteira é comumente encontrada em pequenos pomares, sítios e quintais, com destaque para o Estado de São Paulo, que concentra pomares relativamente maiores (BASTOS, 2004).

Em razão de sua disseminação por mudas oriundas de sementes, apresenta grande variabilidade genética, expressada pela variação do porte, formato da copa, produção, tamanho e qualidade de frutos (LEDERMAN et al., 2000).

A carambola é um fruto atrativo pelo seu formato de estrela, quando cortado em fatias transversais, com casca translúcida e brilhante, polpa amarela de tonalidade intensa e sabor agridoce (NEVES et al., 2004).

As cultivares de caramboleira são classificadas no grupo ácido ou doce, de acordo com a concentração de ácido oxálico na polpa. As cultivares de maior expressão nos países de clima Tropical da América e Ásia são: Golden Star, Arkin, B-10, Fwang Tung e Maha (DONADIO et al., 2001). No Brasil, o Instituto Agronômico de Pernambuco (IPA) lançou a cultivar Cinco Estrelas, que produz cerca de $100 \mathrm{~kg}$ de frutos por planta, nas condições da Zona da Mata de Pernambuco.

A carambola é consumida como fruta fresca, mas a polpa também é utilizada para preparar geleias, molhos, compotas e picles. Essa fruta pode ser enquadrada na categoria de alimentos funcionais ou nutracêuticos, que são aqueles que possuem compostos químicos importantes à saúde humana (KÄHKÖNEN; HEINONEN, 2003; SHUI; LEONG, 2006).

A cor amarela intensa da carambola indica a presença de compostos fitoquímicos, os quais podem exercer propriedades antioxidantes (GROSS et al.,1983; TEIXEIRA et al., 2001), com destaque para carotenoides e fenólicos, que estão relacionados com o retardamento do envelhecimento celular e a prevenção de doenças (HALLIWELL; GUTTERIDGE, 1984; ARTANTI et al., 2006).

Diante do potencial da polpa de carambola na composição de alimentos e bebidas funcionais, este trabalho objetivou avaliar as concentrações de compostos antioxidantes em frutos de acessos de caramboleira do Banco de Germoplasma do Instituto
Agronômico de Pernambuco - IPA, cultivadas em distintos ambientes de Pernambuco.

\section{MATERIAL E MÉTODOS}

O trabalho foi conduzido em três Estações Experimentais do IPA, localizadas nos municípios de Itambé, Ibimirim e Brejão.

O IPA Itambé situa-se no extremo Norte da Zona da Mata de Pernambuco ( $7^{\circ} 24^{\prime} 37^{\prime \prime} \mathrm{S}$ e $35^{\circ} 06^{\prime} 46^{\prime}$ W). O clima é do tipo As' (Köppen), quente e úmido, com temperatura média anual de $25^{\circ} \mathrm{C}$, altitude de $190 \mathrm{~m}$ e pluviosidade média anual de $1.200 \mathrm{~mm}$. O solo predominante é classificado como Argissolo Vermelho-Amarelo.

O IPA Brejão situa-se no Agreste de Pernambuco $\left(09^{\circ} 01^{\prime} 49^{\prime \prime} \mathrm{S}\right.$ e $\left.36^{\circ} 34^{\prime} 07^{\prime \prime} \mathrm{W}\right)$. O clima é do tipo As (Köppen), tropical chuvoso com verão seco, típico de brejo de altitude, com temperatura média anual de $20{ }^{\circ} \mathrm{C}$, altitude de $788 \mathrm{~m}$ e pluviosidade média anual de $900 \mathrm{~mm}$. O solo predominante é classificado como o Argissolo Amarelo.

O IPA Ibimirim está situado no Vale Rio Moxotó, Sertão de Pernambuco ( $8^{\circ} 32^{\prime} 15^{\prime}$ ' S e $37^{\circ}$ 41 '30” W). O clima é do tipo B'Swh' (Köppen), semiárido muito quente, tipo estepe e com temperatura média anual de $28{ }^{\circ} \mathrm{C}$. A altitude é de $431 \mathrm{~m}$, e a pluviosidade média anual, de $420 \mathrm{~mm}$. O solo predominante é classificado como Neossolo Flúvico Eutrófico.

Foram avaliados os frutos dos cinco acessos mais produtivos (IPA-1.3, IPA-7.2, IPA-20.3, IPA-22.3 e IPA-25.1) do Banco de Germoplasma de Caramboleira do IPA, selecionados após 12 anos de caracterização da produção por planta, características físicas do fruto e físico-químicas da polpa.

O Banco de Germoplasma de Caramboleira do IPA foi implantado em 1988 no campo experimental do IPA Itambé. Atualmente, contém 69 acessos, dispostos no espaçamento de 7x7 m e irrigados por microaspersão. A maioria das plantas foi propagada por sementes, coletada em sítios e quintais da Região Metropolitana do Recife, Agreste de Pernambuco e Rio Grande do Norte. Outra parte foi introduzida da UNESP-FCAV/Jaboticabal-SP, e de Israel, por meio de propagação vegetativa.

Os três ensaios para avaliar os frutos dos acessos de carambola foram implantados em 2002. As plantas foram propagadas por enxertia no topo em fenda cheia, utilizando-se de porta-enxertos de sementes da própria planta-matriz. A enxertia foi realizada em porta-enxertos com cerca de 6 meses 
de idade e diâmetro de caule em torno de $5 \mathrm{~mm}$. Os garfos foram retirados da porção mediana de ramos maduros, com aproximadamente 1 ano de idade. As plantas receberam podas de formação e limpeza de copa, adubação de acordo com resultado da análise de solo e irrigadas por microaspersão.

A parcela foi representada por uma planta. Utilizou-se do espaçamento de $7 \times 7 \mathrm{~m}$ e do delineamento experimental inteiramente casualizado, com cinco repetições. Durante a safra de 2008, foram colhidas três amostras de frutos maduros totalmente amarelos, por planta. A amostra foi composta por 20 frutos e acondicionada em caixa isotérmica. As amostras foram transportadas até o Laboratório de Análises Físico-químicas de Alimentos do Departamento de Ciências Domésticas da Universidade Federal Rural de Pernambuco-UFRPE, Recife-PE, onde os frutos foram higienizados e despolpados. A polpa fresca foi acondicionada em sacos de polietileno e armazenada, sob congelamento $\left(-18^{\circ} \mathrm{C}\right)$, até o momento das determinações analíticas: 1) ácido ascórbico (mg.100 g de polpa ${ }^{-1}$ ), determinado numa alíquota de $1 \mathrm{~g}$ de polpa por titulometria, utilizando 2,6 diclorofenol indofenol (AOAC, 2005); 2) carotenoides totais $\left(\mu \mathrm{g} \cdot \mathrm{g}^{-1}\right)$, determinados em $5 \mathrm{~g}$ de polpa maceradas com acetona para extração de pigmentos e transferidos para éter de petróleo por partição (RODRIGUEZ-AMAYA, 1999). Uma alíquota de $10 \mathrm{~mL}$ dos pigmentos extraídos foi aplicada em uma coluna de vidro $(1,5 \times 21 \mathrm{~cm})$, empacotada com celite e óxido de magnésio na proporção de 2:1 até a altura aproximada de $15 \mathrm{~cm}$, tendo no topo aproximadamente $1 \mathrm{~cm}$ de sulfato de sódio anidro. A determinação quantitativa de cada fração foi efetuada considerando sua absorção máxima $\left(\lambda_{\text {máx }}\right)$ e o coeficiente de absorção $\left(\mathrm{E}^{1 \%} \mathrm{~cm}\right)$ . O teor de carotenoides totais foi calculado com base na seguinte expressão matemática: $\mu \mathrm{g} \cdot \mathrm{g}^{-1}=$ Volume $\times \lambda_{\text {máx }} \times 10^{6} / \mathrm{E}^{1 \%} \mathrm{~cm} \times$ peso da amostra; 3$)$ flavonoides (mg.100 g de polpa ${ }^{-1}$ ), determinados por espectrofotometria (DEWANTO et al.,2002). Uma amostra de $70 \mathrm{~g}$ de polpa foi mantida sob agitação por 20 minutos, numa temperatura ambiente de $24^{\circ} \mathrm{C}\left( \pm 2^{\circ} \mathrm{C}\right)$, contendo metanol a $80 \%$ e, em seguida, centrifugada. O sobrenadante foi coletado, o precipitado ressuspenso no mesmo solvente e submetido ao mesmo processo acima descrito, por mais dois períodos de 20 minutos, totalizando 60 minutos de extração. Ao término deste período de extração, sobrenadantes foram combinados, concentrados sob pressão reduzida a $40^{\circ} \mathrm{C}$, e o volume final, aferido para $50 \mathrm{~mL}$. Num balão de $50 \mathrm{~mL}$ foram colocados $5 \mathrm{~mL}$ do extrato, $1,5 \mathrm{~mL}$ de $\mathrm{NaNO} 2$ a $5 \%, 3 \mathrm{~mL}$ de $\mathrm{AlCl} 3$ a $10 \%$, e $10 \mathrm{~mL}$ de $\mathrm{NaOH}$ $1 \mathrm{M}$, e o volume final, aferido com água destilada. A absorbância foi registrada a $510 \mathrm{~nm}$, o teor de flavonoides totais, determinado por interpolação da absorbância das amostras contra uma curva de calibração construída com catequina (20 a 1.600 $\mu \mathrm{g} / \mathrm{mL}$ ); 4) fenólicos totais (mg.g de polpa ${ }^{-1} \mathrm{EAG}$ ), por espectrofotometria, usando reagente de Folinciocalteu (Merck) (WETTASINGHE; SHAHIDI, 1999). O teor de fenóis totais foi determinado por interpolação da absorbância das amostras contra uma curva de calibração construída com ácido gálico (20 a $240 \mu \mathrm{g} / \mathrm{mL}$ ); e 5) taninos condensados (mg.100 g de polpa $^{-1}$ ), de acordo com o método proposto por Tiittojulkunen (1985). O teor de taninos condensados foi determinado por interpolação da absorbância das amostras, registrada a $500 \mathrm{~nm}$, contra uma curva de calibração construída com catequina ( 2 a $40 \mu \mathrm{g} / \mathrm{mL})$.

Os dados foram submetidos às análises de variância individual e conjunta, considerando-se o modelo fixo. Foi verificada a pressuposição de homogeneidade entre as variâncias residuais das análises individuais e ajustados os graus de liberdade da análise conjunta, nos casos em que a relação entre o maior e o menor quadrado médio do resíduo foi superior a sete. As médias foram comparadas pelo teste de Tukey, a 5\% de probabilidade. As análises estatísticas foram realizadas com o auxílio do programa GENES (CRUZ, 2006).

\section{RESULTADOS E DISCUSSÃO}

A significância $(p<0,05)$ para os efeitos de ambiente confirmou que os locais de cultivo são heterogêneos, em relação ao potencial produtivo de frutos com diferentes concentrações médias de ácido ascórbico, flavonóis, fenólicos totais e taninos (Tabela 1). Esses locais representam regiões edafoclimáticas bem definidas de Pernambuco, caracterizadas por solos, temperaturas e precipitações pluviométricas bem distintas entre si. Não foi detectada diferença significativa entre os locais de cultivo para carotenoides totais, indicando que os diferentes ambientes não interferem na concentração desse composto antioxidante nos frutos.

A interação significativa $(p<0,05)$ entre genótipos e ambientes indica que os acessos de caramboleira apresentam respostas diferenciadas às concentrações de ácido ascórbico, flavonóis, fenólicos totais e taninos nos frutos, quando cultivadas em diferentes ambientes (Tabela 1). Essas respostas diferenciadas causam alterações nas ordens de classificação dos melhores acessos nos três ambientes. Não foi detectada diferença significativa 
para interação genótipos e ambientes, em relação a carotenoides totais.

Os acessos de caramboleira diferiram significativamente $(\mathrm{p}<0,05)$ entre $\mathrm{si}$, quanto às concentrações de ácido ascórbico, fenólicos totais e taninos. Não houve diferença significativa entre os acessos para carotenoides totais e flavonoides (Tabela 1).

A concentração de compostos antioxidantes em vegetais pode ser afetada por fatores edafoclimáticos (tipo de solo e condições climáticas), grau de maturação de frutos, constituição genética da planta, efeito de agrotóxicos, condições de processamento e armazenamento da polpa (HUBER; RODRIGUEZ-AMAYA, 2008; SOUZA et al., 2011).

As médias de ácido ascórbico nos frutos variaram de 2,14 a 20,62 mg.100 g de polpa ${ }^{-1}$. Esses resultados foram inferiores àqueles apresentados por Teixeira et al. (2001) com variações de 12,54 a 26,28 mg.100 g de polpa-1 (Tabela 2).

As médias para carotenoides totais oscilaram entre 0,31 e 3,87 $\mu \mathrm{g} \cdot \mathrm{g}^{-1}$. Esses valores estão dentro da variação de 0,66 a $0,91 \mu \mathrm{g} \cdot \mathrm{g}^{-1}$ apresentada por Souza et al. (2004) e muito abaixo da média de 7,50 $\mu \mathrm{g} \cdot \mathrm{g}^{-1}$ apresentada por Gross et al. (1983).

A concentração média para flavonóis variou de 2,94 a 5,77 mg.100 g de polpa ${ }^{-1}$. Os fenólicos totais atingiram médias entre 21,74 e 85,33 mg. 100 g de polpa ${ }^{-1}$ EAG. A concentração de taninos variou de 138,25 a $11.010,00 \mathrm{mg} .100 \mathrm{~g}$ de polpa ${ }^{-1}$. A maior concentração de ácido ascórbico foi verificada nos frutos do acesso IPA-7.2, cultivados em Brejão (20,78 mg.100 g de polpa $\left.{ }^{-1}\right)$ e Ibimirim (19,48 mg.100 g de polpa $\left.^{-1}\right)$, superando significativamente $(\mathrm{p}<0,05)$ os outros acessos dentro de cada local, respectivamente. Em Itambé, o acesso IPA-7.2 apresentou a maior média de 17,49 mg.100 g de polpa ${ }^{-1}$, que superou significativamente os demais acessos.

Os frutos dos acessos IPA-22.3 e IPA-1.3, cultivados em Itambé, apresentaram as maiores médias de 3,87 e 3,86 $\mu \mathrm{g}$. $\mathrm{g}^{-1}$ para carotenoides totais, respectivamente. Contudo, as mesmas não diferiram significativamente $(\mathrm{p}<0,05)$ dos acessos IPA-7.2 e IPA-25.1. Em Brejão, os frutos do acesso IPA-1.3 atingiram a maior média de $3,84 \mu \mathrm{g} . \mathrm{g}^{-1}$, superando significativamente os demais acessos. Em Ibimirim, os frutos do acesso IPA-22.3 atingiram 3,71 $\mu \mathrm{g} . \mathrm{g}^{-1}$, não diferindo significativamente dos acessos IPA20.3 e IPA-7.2.

Os acessos IPA-22.3, IPA-25.1, IPA-7.2 e IPA-20.3, cultivados em Itambé, não diferiram significativamente $(\mathrm{p}<0,05)$ entre si com relação à concentração dos flavonoides. O acesso IPA22.3, cultivado em Ibimirim, atingiu a média de
5,57 mg de flavonóis.100 g de polpa-1, superando significativamente os outros acessos, com exceção do IPA-20.3.

As maiores médias de 85,33 e 78,30 mg de fenólicos totais. $100 \mathrm{~g}$ de polpa $^{-1}$ foram obtidas nos frutos dos acessos IPA-22.3 e IPA-25.1, cultivados em Ibimirim e Itambé, respectivamente. As mesmas superaram significativamente $(\mathrm{p}<0,05)$ os outros acessos, dentro de cada local de cultivo.

Em Itambé, os frutos do acesso IPA-22.3 apresentaram a maior média de $11.010 \mathrm{mg}$ de taninos. $100 \mathrm{~g}$ de polpa $^{-1}$, superando significativamente $(\mathrm{p}<0,05)$ os demais acessos.

Considerando as médias dos acessos, os frutos da caramboleira IPA-7.2 apresentaram 19,25 $\mathrm{mg}$ de ácido ascórbico. $100 \mathrm{~g}$ de polpa $^{-1}$, superando significativamente $(\mathrm{p}<0,05)$ os demais acessos. Quanto às concentrações de fenólicos totais e taninos, os frutos do acesso IPA-22.3 atingiram as respectivas médias de 64,59 e 4.096,99 mg.100 g de polpa $^{-1}$, superando significativamente os outros acessos. Para carotenoides totais e flavonóis, os frutos da caramboleira IPA-22.3 também apresentaram as maiores médias de 3,07 $\mu \mathrm{g} \cdot \mathrm{g}^{-1}$ e 4,82 mg.100 g de polpa $^{-1}$, respectivamente, sem haver diferença significativa em relação aos demais acessos.

Quanto às médias dos ambientes, verificamos que as condições de cultivo do IPA em Itambé superaram significativamente $(\mathrm{p}<0,05)$ as demais, proporcionando aos frutos as maiores concentrações de ácido ascórbico (11,53 mg.100 g de polpa-1), fenólicos totais $\left(63,74 \mathrm{mg} .100 \mathrm{~g}\right.$ de polpa $\left.{ }^{-1} \mathrm{EAG}\right) \mathrm{e}$ taninos $\left(3.722,05 \mathrm{mg} .100 \mathrm{~g}^{\mathrm{de}}\right.$ polpa $\left.^{-1}\right)$. Não houve diferença significativa entre os locais de cultivo para carotenoides totais, que variaram de 2,78 (IPA Itambé) a 1,53 $\mu \mathrm{g} \cdot \mathrm{g}^{-1}$ (IPA Brejão). As médias para flavonóis, nos ambientes de Itambé $(5,17 \mathrm{mg} .100 \mathrm{~g}$ de polpa $\left.^{-1}\right)$ e de Ibimirim (4,50 mg. $\left.100 \mathrm{~g} \mathrm{de} \mathrm{polpa}^{-1}\right)$ não diferiram significativamente entre si. Contudo, esses locais superam a média de 3,28 mg de flavonóis. 100 $\mathrm{g}$ de polpa $^{-1}$, obtida nas condições de cultivo do IPA Brejão. 
TABELA 1- Resumo da análise de variância conjunta dos teores médios de ácido ascórbico, carotenoides totais, flavonóis, fenólicos totais e taninos em frutos de cinco acessos de carambola (Averrhoa carambola), cultivadas nos municípios de Itambé, Ibimirim e Brejão, localizados na Zona da Mata, Sertão e Agreste de Pernambuco, respectivamente.

\begin{tabular}{|c|c|c|c|c|c|c|}
\hline \multirow[b]{2}{*}{ Características } & \multirow[b]{2}{*}{ GL } & \multicolumn{5}{|c|}{ Quadrados médios } \\
\hline & & $\begin{array}{c}\text { Ácido } \\
\text { ascórbico }\end{array}$ & Carotenóides totais & Flavonóis & Fenólicos totais & Taninos \\
\hline Ambientes (A) & 2 & $69,53^{*}$ & $6,72^{\text {ns }}$ & $13,74 *$ & $2.578,01^{*}$ & $38.265 .329,73^{*}$ \\
\hline Genótipos (G) & 4 & $303,46^{*}$ & $6,43^{\mathrm{ns}}$ & $1,28^{\mathrm{ns}}$ & $748,94 *$ & $17.063 .718,17 *$ \\
\hline $\mathrm{G} \times \mathrm{A}$ & 8 & $46,05^{*}$ & $3,88^{\mathrm{ns}}$ & $0,94 *$ & $1.136,19 *$ & $25.336 .547,21 *$ \\
\hline Resíduo & 30 & 0,87 & 0,61 & 0,26 & 18,10 & $25.296,77$ \\
\hline$\overline{\mathrm{CV}(\%)^{1}}$ & & 10,31 & 38,85 & 11,83 & 8,39 & 8,11 \\
\hline Média geral & & 9,05 & 2,01 & 4,31 & 50,66 & $1.961,64$ \\
\hline
\end{tabular}

* significativo a $5 \%$ de probabilidade, pelo teste $\mathrm{F}$.

TABELA 2- Concentrações médias de ácido ascórbico, carotenoides totais, flavonóis, fenólicos totais e taninos nos frutos de cinco acessos de caramboleira (Averrhoa carambola), cultivadas nos municípios de Itambé, Ibimirim e Brejão, localizados na Zona da Mata, Sertão e Agreste de Pernambuco, respectivamente.

\begin{tabular}{|c|c|c|c|c|}
\hline \multirow{3}{*}{ Acessos } & \multicolumn{3}{|c|}{ Ambientes } & \multirow{2}{*}{ Médias } \\
\hline & Itambé & Ibimirim & Brejão & \\
\hline & \multicolumn{4}{|c|}{ 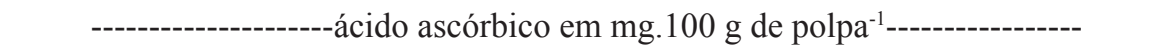 } \\
\hline IPA-1.3 & $3,31 \mathrm{~dB}$ & $8,46 \mathrm{bA}$ & $2,15 \mathrm{~dB}$ & $4,64 \mathrm{c}$ \\
\hline IPA-7.2 & $17,49 \mathrm{aB}$ & $19,48 \mathrm{aA}$ & $20,78 \mathrm{aA}$ & $19,25 \mathrm{a}$ \\
\hline IPA-20.3 & $14,66 \mathrm{bA}$ & $2,14 \mathrm{~dB}$ & $3,67 \mathrm{cdB}$ & $6,82 \mathrm{bc}$ \\
\hline IPA-22.3 & $10,52 \mathrm{cA}$ & $4,02 \mathrm{cdC}$ & $6,58 \mathrm{bB}$ & $7,04 \mathrm{~b}$ \\
\hline IPA-25.1 & $11,70 \mathrm{cA}$ & $5,64 \mathrm{cB}$ & $5,19 \mathrm{bcB}$ & $7,51 \mathrm{~b}$ \\
\hline \multirow[t]{2}{*}{ Médias } & $11,53 \mathrm{~A}$ & $7,95 \mathrm{~B}$ & $7,67 \mathrm{~B}$ & \\
\hline & & carotenoide & $\mu \mathrm{g} \cdot \mathrm{g}^{-1}$ & \\
\hline IPA-1.3 & $3,86 \mathrm{aA}$ & $0,35 \mathrm{bB}$ & $3,84 \mathrm{aA}$ & $2,68 \mathrm{a}$ \\
\hline IPA-7.2 & $2,70 \mathrm{abA}$ & $1,68 \mathrm{abA}$ & $1,32 \mathrm{bA}$ & $1,90 \mathrm{a}$ \\
\hline IPA-20.3 & $1,53 \mathrm{bA}$ & $1,99 \mathrm{abA}$ & $0,31 \mathrm{bA}$ & $1,28 \mathrm{a}$ \\
\hline IPA-22.3 & $3,87 \mathrm{aA}$ & $3,71 \mathrm{aA}$ & $1,62 \mathrm{bB}$ & $3,07 \mathrm{a}$ \\
\hline IPA-25.1 & $1,93 \mathrm{abA}$ & $0,97 \mathrm{bA}$ & $0,54 \mathrm{bA}$ & $1,15 \mathrm{a}$ \\
\hline Médias & $2,78 \mathrm{~A}$ & $1,74 \mathrm{~A}$ & $1,53 \mathrm{~A}$ & \\
\hline & \multicolumn{4}{|c|}{ 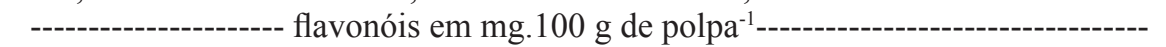 } \\
\hline IPA-1.3 & $4,02 \mathrm{bAB}$ & $4,31 \mathrm{bA}$ & $3,14 \mathrm{aB}$ & $3,82 \mathrm{a}$ \\
\hline IPA-7.2 & $5,30 \mathrm{aA}$ & $4,19 \mathrm{bB}$ & $4,02 \mathrm{aB}$ & $4,50 \mathrm{a}$ \\
\hline IPA-20.3 & $5,18 \mathrm{abA}$ & $4,53 \mathrm{abA}$ & $3,15 \mathrm{aB}$ & $4,28 \mathrm{a}$ \\
\hline IPA-22.3 & $5,77 \mathrm{aA}$ & $5,57 \mathrm{aA}$ & $3,14 \mathrm{aB}$ & $4,82 \mathrm{a}$ \\
\hline IPA-25.1 & $5,57 \mathrm{aA}$ & $3,88 \mathrm{bB}$ & $2,94 \mathrm{aB}$ & $4,13 \mathrm{a}$ \\
\hline \multirow[t]{2}{*}{ Médias } & $5,17 \mathrm{~A}$ & $4,50 \mathrm{~A}$ & $3,28 \mathrm{~B}$ & \\
\hline & 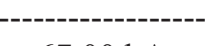 & licos totais & $\mathrm{g}$ de polpa $^{-1}$ & ---------- \\
\hline IPA-1.3 & $67,00 \mathrm{bA}$ & $39,23 \mathrm{cB}$ & $21,74 \mathrm{dC}$ & $42,66 \mathrm{c}$ \\
\hline IPA-7.2 & $67,71 \mathrm{bA}$ & $63,02 \mathrm{bA}$ & $33,08 \mathrm{bcB}$ & $54,60 \mathrm{ab}$ \\
\hline IPA-20.3 & $62,18 \mathrm{bA}$ & $41,08 \mathrm{cB}$ & $27,00 \mathrm{cdC}$ & $43,42 \mathrm{c}$ \\
\hline IPA-22.3 & $43,50 \mathrm{cC}$ & 85,33 aA & $64,93 \mathrm{aB}$ & $64,59 \mathrm{a}$ \\
\hline IPA-25.1 & $78,30 \mathrm{aA}$ & $24,99 \mathrm{dC}$ & $40,84 \mathrm{bB}$ & $48,04 \mathrm{bc}$ \\
\hline \multirow[t]{2}{*}{ Médias } & $63,74 \mathrm{~A}$ & $50,73 \mathrm{~B}$ & $37,52 \mathrm{C}$ & \\
\hline & \multicolumn{4}{|c|}{ 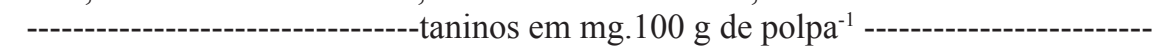 } \\
\hline IPA-1.3 & $138,25 \mathrm{~dB}$ & $520,00 \mathrm{abA}$ & $739,28 \mathrm{cdA}$ & $465,84 \mathrm{e}$ \\
\hline IPA-7.2 & $1.759,67 \mathrm{cB}$ & $838,73 \mathrm{aC}$ & $2.482,25 \mathrm{bA}$ & $1693,55 \mathrm{c}$ \\
\hline IPA-20.3 & $5.477,00 \mathrm{bA}$ & $465,80 \mathrm{abC}$ & $1.089,83 \mathrm{cB}$ & $2.344,21 \mathrm{~b}$ \\
\hline IPA-22.3 & $11.010,00 \mathrm{aA}$ & $811,63 \mathrm{aB}$ & $469,33 \mathrm{dC}$ & $4.096,99 \mathrm{a}$ \\
\hline IPA-25.1 & $225,33 \mathrm{~dB}$ & $390,10 \mathrm{bB}$ & $3.007,49 \mathrm{aA}$ & $1.207,64 \mathrm{~d}$ \\
\hline Médias & $3.722,05 \mathrm{~A}$ & $605,25 \mathrm{C}$ & $1.557,64 \mathrm{~B}$ & \\
\hline
\end{tabular}

Médias seguidas pelas mesmas letras, minúsculas na coluna e maiúsculas na linha, não diferem estatisticamente entre si, pelo teste de Tukey, com $5 \%$ de probabilidade. 


\section{CONCLUSÕES}

As condições de cultivo de Itambé proporcionaram aos frutos as maiores concentrações de ácido ascórbico, fenólicos totais e taninos. Tanto o ambiente de Itambé, quanto o Ibimirim proporcionaram aos frutos os maiores teores de flavonoides totais. Para carotenoides totais, não houve diferença significativa entre os distintos locais de cultivo.

Os frutos do acesso de caramboleira IPA7.2 apresentaram a maior concentração de ácido ascórbico. Para fenólicos totais e taninos, os frutos do acesso IPA-22.3 atingiram as médias mais altas. Quanto às concentrações de carotenoides totais e flavonóis, não houve diferenças significativas entre os frutos dos acessos de caramboleira.

\section{REFERÊNCIAS}

AOAC - Association of Official Analitycal Chemists. Official methods of analysis of AOAC Internacional. $18^{\text {th }}$ ed. Gaithsburg: AOAC, 2005.

ARTANTI, N. M. Y.; HANAFI, M. Isolation and Identification of Active Antioxidant Compound from Star Fruit (Averrhoa carambola) Mistletoe (Dendrophthoe pentandra Ethanol Extract. Journal of Applied Sciences, Pakistan, v.6, n.8, p. 16591663, 2006.

BASTOS, D. C. A cultura da carambola. Revista Brasileira de Fruticultura, Jaboticabal, v. 26, n. 2, p.193-384, 2004.

CRUZ, C. D. Programa Genes: biometria. Viçosa: Editora UFV, 2006. 384 p.

DEWANTO, V.; WU, X.; ADOM, K. K.; LIU, R. H. Thermal processing enhances the nutritional value of tomatoes by increasing total antioxidant activity. Journal of Agriculture and Food Chemical, Davis, v. 50, n.10, p.3010-3014, 2002.

DONADIO, L. C.; SILVA, J. A. A.; ARAÚJO, P. S. R.; PRADO, R. M. Caramboleira (Averrhoa carambola L.) Revista Brasileira de Fruticultura, Jaboticabal, v.33, n.4, p.1300-1307, 2001.

GROSS, J.; IKAN, R.; ECKHARDT, G. Carotenoids of the fruit of Averrhoa carambola. Phytochemistry, New York, v.22, n.6, p.1479-1481, 1983.
HALLIWELL, B.; GUTTERIDGE, J. M. Oxygen toxicity, oxygen radicals, transition metals and disease. Biochemical Journal, London, v.219, n.1, p.1-14. 1984.

HUBER, L.S.; RODRIGUEZ-AMAYA, D.B. Flavonóis e flavonas: fontes brasileiras e fatores que influenciam a composição em alimentos. Alimentos e Nutrição, Campinas, v.19, n.1, p.97-108, 2008.

KÄHKÖNEN, M. P.; HEINONEN, M. Antioxidant activity of anthocyanins and their aglycons. Journal of Agricultural and Food Chemistry, Davis, v.51, n.3, p.628-633, 2003.

LEDERMAN, I. E.; BEZERRA, J. E. F.; ASSUNÇÃO, M. A.; FREITAS, E. V. Caracterização e seleção de genótipos de caramboleiras (Averrhoa carambola L.) em Pernambuco. Revista Brasileira de Fruticultura, Jaboticabal, v. 22, n. 1, p.3135. 2000 .

NEVES, L. C.; BENDER, R. J.; ROMBALDI, C. V.; VIEITES, R. L. Qualidade de carambolas azedas cv. 'Golden Star' tratadas com $\mathrm{CaCl}_{2}$ por imersão e armazenadas sob refrigeração. Revista Brasileira de Fruticultura, Jaboticabal, v.26, n.1, p.102123, 2004.

RODRIGUEZ-AMAYA, D. B. A guide to carotenoid analysis in foods. Washington: ILSI Press, 1999. 64 p.

SHUI, G.; LEONG, L. P. Residue from starfruit as valuable source for functional food ingradients and antioxidant neutraceuticals. Food Chemistry, Oxford, v. 97, n.2, p.277-284, 2006.

SOUSA, M. S. B.; VIEIRA, L. M.; SILVA, M. de J. M. da; LIMA, A. de. Caracterização nutricional e compostos antioxidantes em resíduos de polpas de frutas tropicais. Ciência Agrotecnologia, Lavras, v. 35, n. 3, p. 554-559, 2011.

SOUZA, S. L.; MOREIRA, A. P. B.; PINHEIROSANT'ANA H. M.; ALENCAR, E. R. Conteúdo de carotenos e provitamina A em frutas comercializadas em Viçosa, Estado de Minas Gerais. Acta Scientiarum Agronomy, Maringá, v. 26, n. 4, p. 453-459, 2004. 
TEIXEIRA, G. H. A.; DURIGAN, J. F.; DONADIO, L. C.; SILVA, J. A. A. Caracterização pós-colheita de seis genótipos de carambola (Averrhoa carambola L.). Revista Brasileira de Fruticultura, Jaboticabal, v.24, n.1, p.546-550, 2001.

TIITTO-JULKUNEN, R. Phenolic constituents in the leaves of Northern Willows: Methods for the analysis of certain phenolics. Journal of Agricultural and Food Chemistry, Davis, v. 33, n. 2, p. 213-217, 1985.
WETTASINGHE, M.; SHAHIDI, F. Evening primrose meal: a source of natural antioxidants and scavenger of hydrogen peroxide and oxygen-derived free radicals. Journal of Agricultural and Food Chemistry, Washington, v. 47, n. 5, p.1801-1812, 1999. 